\title{
A Hands-Free Obstacle Detection and Avoidance System
}

\author{
Mohammed Abdul-Niby \\ School of Engineering \\ The Australian College of Kuwait, Kuwait \\ m.nibi@ack.edu.kw
}

\author{
Mohamad Baidoun \\ School of Engineering, The \\ Australian College of Kuwait, \\ Kuwait \\ m-baidoun@hotmail.com
}

\author{
Mamoon Alameen \\ School of Engineering \\ The Australian College of Kuwait, Kuwait \\ m.radiy@ack.edu.kw
}

\author{
Otmar Irscheid \\ School of Engineering \\ Kuwait \\ o.irscheid@ack.edu.kw
}

\begin{abstract}
In this paper, we present a low cost hands-free detection and avoidance system designed to provide mobility assistance for visually impaired people. An ultrasonic sensor is attached to the jacket of the user and detects the obstacles in front. The information obtained is transferred to the user through audio messages and also by a vibration. The range of the detection is user-defined. A text-to-speech module is employed for the voice signal. The proposed obstacle avoidance device is cost effective, easy to use and easily upgraded.
\end{abstract}

Keywords-intelligent navigation capabilities; electronic travel aids; ultrasonic sensor

\section{INTRODUCTION}

Vision is probably the most important sense for a human body, as it gets about $83 \%$ of its information through sight. Statistics by the World Health Organization estimates that there are 285 million people in the world with visual impairment, 39 million of which are blind and 246 million are with low vision [1]. The oldest mobility aids for people with visual impairments are walking canes (sticks) and guide dogs. The most important drawbacks of these aids are the training needed, the range of motion and that the information conveyed is very little. Also, that they do fairly little to counter the main problem that visually impaired people face: walking or moving in an unfamiliar area, with no prediction regarding the surrounding environment's obstacles and obstructions. This results to both a psychological as well as a practical dependence from third-party assistance.

Nowadays, a great variety of improvements in hardware and software technologies may provide added help through the use of intelligent navigation capabilities. Recently, there has been a wide variety of Electronic Travel Aids (ETA) designed and developed in order to make navigation easier and help visually impaired people to live their lives safely and independently. Different aids have been developed, two of the most significant are listed below:
- Ultracane by Sound Foresight Technology Ltd: The Ultracane is equipped with a dual-range, narrow beam ultrasound system that provides a $100 \%$ hazard protection envelope in front of and, uniquely, forward of the head and chest of the user. Two ultrasound transducers provide range data on the closest potential hazards, such as plants, people, road signs, and over-hanging branches [2].

- Mowat Sensor: a lightweight, hand-held device which has similar properties to a flashlight. The sensor itself detects objects by measuring the distance of a vibration of the brief pulses of HF sound it emits. The device will then inform the person how close an object may be [3]

However, these techniques focus on outdoor movement. They can obviously be used indoors but, for example, the cane can be rather disturbing indoors. On the other hand, the Mowat sensor must be placed in a pocket in order to keep contact with the body so that the vibration is felt by the user.

The goals set for an improved system sums up to designing a system that can help the user to navigate indoors and outdoors in such a way that:

- it is hands free and easy to use

- $\quad$ can be used by users of different ages

- doesn't interfere with the user's daily behavior/way of living.

At the same time, the design should overcome problems such as reliability, cost, availability, high cost and flexibility/upgradeability.

\section{SYSTEM DESCRIPTION}

The system described in this paper aims to help visually impaired people secure their path and experience a safer way of living. It uses ultrasound detection and warns the user via a voice 
signal. Also, taking into consideration that some users might have a hearing problem, it vibrates once an object is detected.

\section{A. Basic operation}

The device main stages of operation can be summarized to the four stages shown in Figure 1. Once an object is detected by the ultrasonic sensor, a signal is sent to the processor to inform that there is an object ahead, then the processor turns on the two outputs and thus the user hears the message and senses the vibration. A state diagram [4] is shown in Figure 2 [4] whereas Table I shows the truth table for the device. The sequence of the device operation is shown in Figure 3. As shown, the device is initially at idle state, where it is just sensing and waiting for a signal to be detected. It moves to the next state, which is "receive", when it detects a signal ahead. Then it moves to the next state ("alert"), whose output is the activation of the two alerts present in the system, the vibrating motor, and the voice chip.

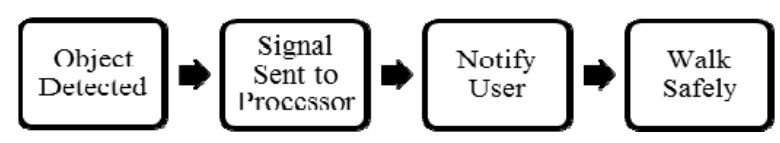

Fig. 1. Block Diagram for the General System Functions.

TABLE I. THE TRUTH TABLE FOR THE DEVICE

\begin{tabular}{|c|c|c|c|}
\hline Current State & Input* $^{*}$ Next State & Output \\
\hline \multirow{2}{*}{ Idle } & 0 & Idle & None \\
\cline { 2 - 4 } & 1 & Receive & Get Signal \\
\hline \multirow{2}{*}{ Receive } & 0 & Idle & None \\
\cline { 2 - 4 } & 1 & Alert & Output is true \\
\hline \multirow{2}{*}{ Alert } & 0 & Idle & None \\
\cline { 2 - 4 } & 1 & Alert & Voice \& vibration ON \\
\hline
\end{tabular}

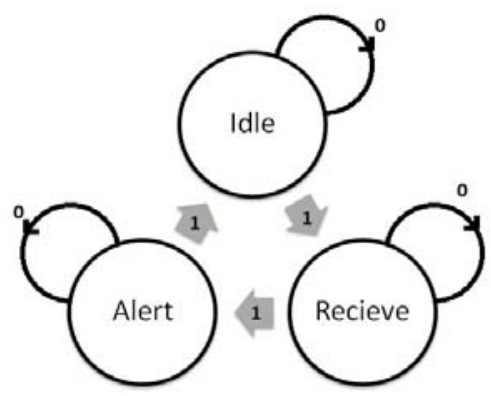

Fig. 2. The State Diagram

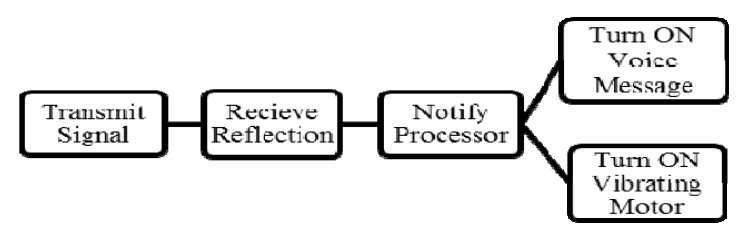

Fig. 3. The Sequence of the Device Operation
The ultrasound signal, which is a sound signal above the human hearing capability is generated and transmitted by the ultrasound transceiver [5]. This transceiver transmits and receives ultrasound signals at the same time. Once an ultrasonic signal propagates, it continues traveling through air until it is obstructed by an object. The signal then bounces off the obstacle and is reflected back towards the transceiver. At this stage, the transceiver detects the reflected signal; once this happens it sends a pulse to the processor's input notifying it that an object has been detected.

When the processor gets the input from the sensor, it is preprogrammed so that it turns on two outputs, which are simply two pins each connected to a distinct circuit. One pin connects to the voice playback circuit, while the other is connected to the vibrating motor. These pins can give pulses (logic outputs), and the design configuration is done such that the two alerting circuits, which are the voice message playback circuit and the vibrating motor, are activated in order to notify the user by playing a verbal message and generating a physical vibration within the box which will be mounted on the user's waist.

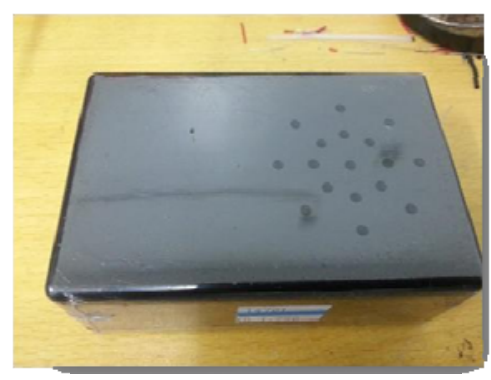

Fig. 4. Processor Box

\section{B. Device Design}

The shape of the device is very simple and easy to implement or install by the user. A box containing the circuitry (Figure 4), i.e. the processor, the voice message playback circuit and the vibrating motor, will be mounted on the user's waist using a clipper. Mounting the box on the waist will allow the user to feel the vibration. The transceiver (Figure 5) is attached to a clipper that can be mounted on different heights of the body for flexibility of use. Also, an earphone or speaker jack input is available at the back on the box that can be used to connect different kind of headsets.

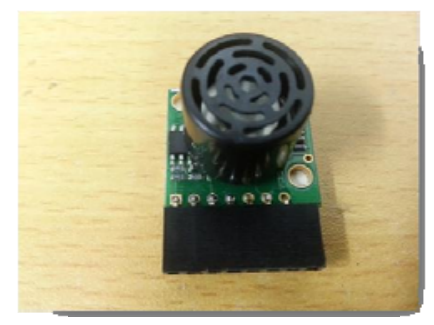

Fig. 5. Ultrasound Transceiver 
The Arduino Uno-R3 SMD board was used in the design of the system because of its high capability of controlling inputs and outputs, due to it being based on the ATmega328 Atmel ${ }^{\circledR}$ board [6]; it can be programmed easily using AVR ${ }^{\circledR}$ studio software provided by Atmel®. The programming language used is $\mathrm{C}$ that the compiler translates to assembly.

\section{Emic2 Text to Speech Module [7]}

This module (shown in Figure 6) is a multi-language voice synthesizer that converts a stream of digital text into natural sounding speech. It makes it simple to convert a string of text to audible voice text using the universally recognized DECtalk text-to-speech synthesizer engine. A visual indication of the operating state is given with an on-board LED light as shown in Table II [7]. An image of the Emic2 board and a schematic diagram, are shown in Figure 6.

TABLE II. VISUAL INDICATION OF THE OPERATING STATE

\begin{tabular}{|c|c|}
\hline Colour & State \\
\hline Green & Idle State \\
\hline Red & Active State \\
\hline Orange (Solid) & Initialization State \\
\hline Orange (Blinking) & Error State \\
\hline LED OFF & Device is not receiving \\
\hline
\end{tabular}

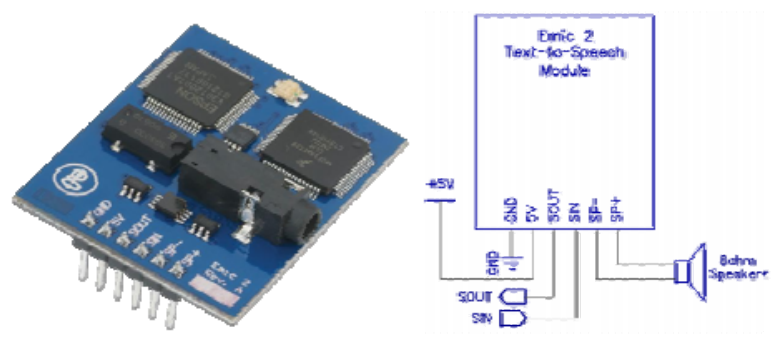

Fig. 6. Emic2 Board and its Schematic Diagram

\section{Ultrasonic Range Finder LV-EZ1 [8]}

LV-EZ1 provides very short to long-range detection (Figure 5). It can detect objects from 0 -inches to 254-inches (6.45 meters) and provides sonar range information from 6inches out to 254-inches with 1-inch resolution. The interface output formats included are pulse width output, analog voltage output and serial digital output.

\section{E. Vibrating Motor}

The ROB-08449 vibration motor (Figure 7) is a tiny shaftless vibratory motor which is perfect for non-audible indicators. It can be used in different applications to indicate an event [9].

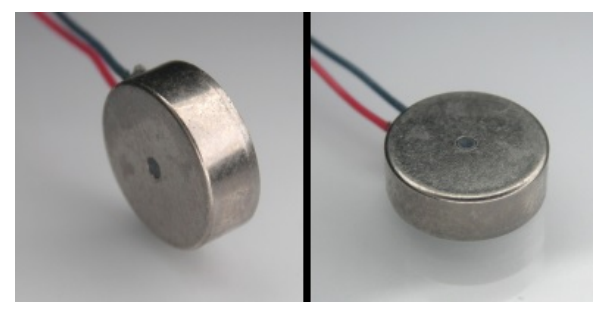

Fig. 7. The ROB-08449 Vibration Motor
All moving parts are protected within the housing. With a $2-3.6 \mathrm{~V}$ operating range, these units shake crazily at $3 \mathrm{~V}$. Once anchored to a printed circuit board (PCB) or within a pocket, the unit vibrates softly but noticeably. This high quality unit comes with a 3M adhesive backing and reinforced connection wires.

\section{F. Power Source}

The system uses multi-voltage power sources, that provide each component with its corresponding power, i.e.:

- The Arduino board requires 7-12 volts.

- $\quad$ The Ultrasonic Sensor requires 2.5-5.5 volts.

- $\quad$ The Emic 2 playback circuit requires 5volts.

- $\quad$ The Vibrating motor requires 3 volts.

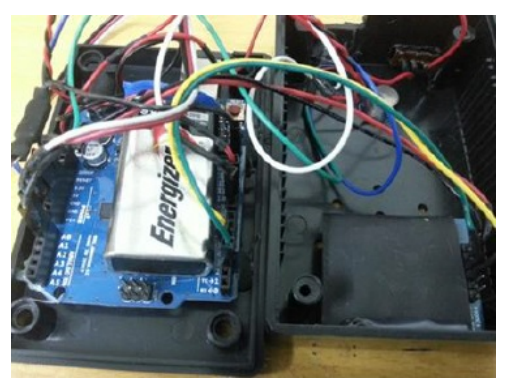

Fig. 8. Installation of the Power Source and the Connections

\section{SYSTEM OPERATION IN DETAIL}

The ultrasound sensor has three wires connected to it, one for the +5 volts in red color that power the sensor, a black wire connecting the GND pin to common ground, and a white wire connecting the sensor to the input of the Arduino. This white wire is connected to the PW pin of the LV-EZ1 ultrasound sensor. This pin is responsible for sending a pulse with an amplitude of +5 volts to the input of the microcontroller, which is picked up by the input pin of the Arduino as a logic 1 . The Arduino is programmed in such a way that once a logic 1 is received, the two outputs that are connected to the Emic2 and the vibrating motor are also set to logic 1 . This activates the Emic2 and also provides power to the vibrating motor. The Emic2 receives the logic 1 via the " $S_{\text {in }}$ " pin which is the pin responsible for receiving an input signal that will activate the verbal message. The verbal message will be played via the headset which will be connected to the jack connector.

During the programming of the Emic2, a suitable was input in the code, and the written text will be played as voice. Meanwhile, the vibrating motor will be switched on, powered by the logic 1 given by the Arduino. This process is repeated each time an obstacle is detected by the ultrasound sensor. Noting that the outputs will remain switched on as long as the obstacle is present. Figure 9 shows the practical connection between the different circuitry. This hands-free obstacle detection and avoidance system was set and tested to work in a range of approximately 1.5 meters. On the box, next to the ONOFF switch, there is a potentiometer that controls the sensor's range. The maximum range of the sensor is 10 meters. 


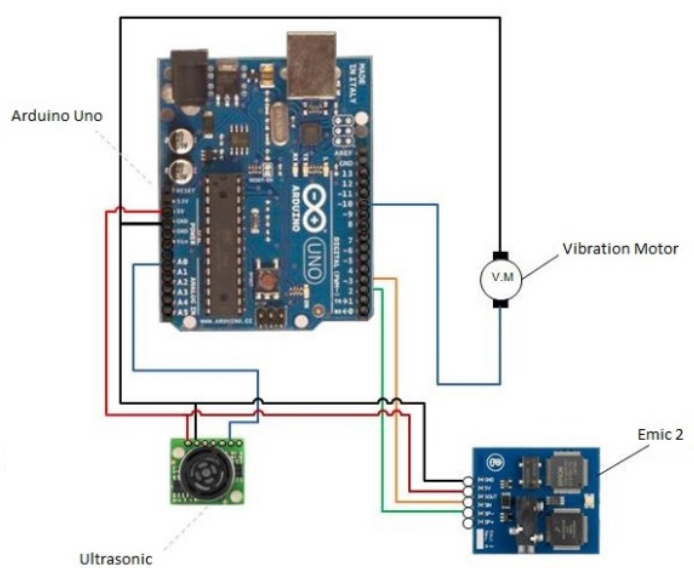

Fig. 9. Practical connection between the different circuitry

\section{CONCLUSION AND FUTURE WORK}

The obstacle detection described in this paper provides a low cost, easy to use flexible system that can be used to aid the movement of the visually impaired. The main idea behind the system is to use an ultrasound detection system in collaboration with a voice notification system. Several improvements that where added to the main idea such as the vibration notification, the adjustable range of the detector and the use of a text-tospeech module underline the flexibility of the approach. The main advantages of the system is the simplicity of the design, and thus its low-cost and upgrade possibilities, the ease of operation and hands free capability. Its main weaknesses currently are that it was not designed for a rechargeable power source and that it is not weather proof.

Further improvement can be employed:

- $\quad$ adding a rechargeable power source.

- $\quad$ adding more than one ultrasound sensor that can be placed at different heights of the human body to secure and cover a wider range of angles and different directions.

- $\quad$ adding guidance, such that the system informs the user if the object is on the left side, right side, lower level and/or higher level.

- reduce the size and weight of the device by building the different circuits on a single printed circuit board and using a small sized casing made of low weight material.

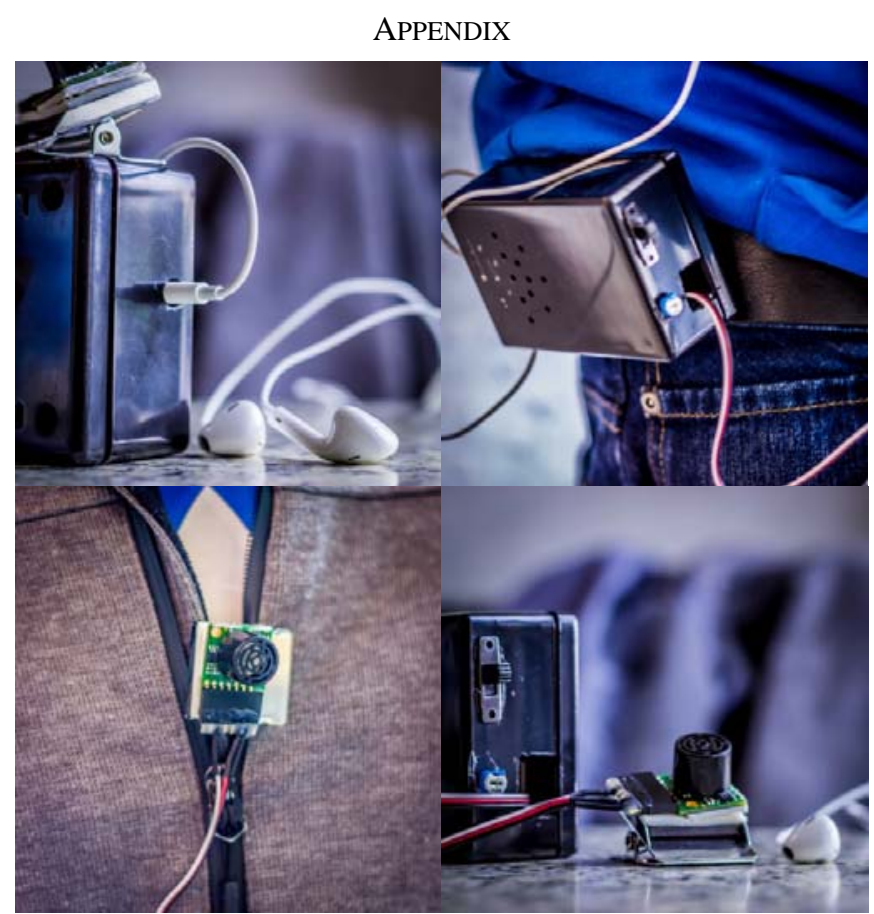

\section{REFERENCES}

[1] D. Pascolini, S. P. Mariotti, "Global estimates of visual impairment: 2010”, British Journal of Ophthalmology, Vol. 96, No. 5, pp. 614-618, 2012

[2] http://www.ultracane.com/about_the_ultracane

[3] http://psychologydictionary.org/mowat-sensor

[4] http://www.techopedia.com/ definition/16446/state-diagram

[5] Ultrasonic Transducers Technical Notes, Olympus, 2006 (available at: www.olympus-ims.com/data/File/panametrics/UT-technotes.en.pdf)

[6] http://arduino.cc/en/Main/arduinoBoardUno

[7] Emic 2 Text-to-Speech Module (\#30016), Parallax inc, 2012 (available through: Mouser Electronics Website http://www.mouser. com/ds/2/321/30016-Emic2TextToSpeech-v1.1-27211.pdf)

[8] LV-EZ1MaxbotixUltrasonic, Rangefinder, http://www.adafruit.com/ products/172

[9] Sparkfun.com. 2009. Vibration Motor-ROB-08449-SparkFun Electronics (available at: https://www.sparkfun.com/ products/8449) 